\title{
Optical Input/Electrical Output Memory Elements Based on a Liquid Crystalline Azobenzene Polymer
}

Thomas Mosciatti, ${ }^{a, b}$ Sara Bonacchi, ${ }^{a}$ Marco Gobbi, ${ }^{a}$ Laura Ferlauto, ${ }^{a, c}$ Fabiola Liscio, ${ }^{c}$ Loris

Giorgini, ${ }^{d}$ Emanuele Orgiu, ${ }^{a}$ and Paolo Samori ${ }^{*}, a$.

a. $\quad$ ISIS \& icFRC, Université de Strasbourg \& CNRS, 8 allée Gaspard Monge, 67000 Strasbourg, France

b. Scriba Nanotecnologie, via di Corticella 183/8, 40128 Bologna, Italy.

c. Istituto per la Microelettronica e Microsistemi (IMM)-CNR, via Piero Gobetti 101, 40129 Bologna, Italy.

d. Dipartimento Chimica Industriale Toso Montanari, Università di Bologna, Viale Risorgimento 4, 40136 Bologna, Italy.

KEYWORDS: Memory device, azobenzene, photochromic polymers, polymeric liquid crystals, multifunctional device.

ABSTRACT: Responsive polymer materials can change their properties when subjected to external stimuli. In this work, thin films of thermotropic poly(metha)acrylate/azobenzene polymers are explored as active layer in light-programmable, electrically-readable memories. The memory effect is based on the reversible modifications of the film morphology induced by the photo-isomerization of azobenzene mesogenic groups. When the film is in the liquid crystalline phase, the trans $\rightarrow$ cis isomerization induces a major surface reorganization on the mesoscopic scale that is characterized by a reduction in the effective thickness of the film. The film conductivity is measured in vertical two-terminal devices in which the polymer is sandwiched between an Au contact and a liquid compliant E-GaIn drop. We demonstrate that the 
trans $\rightarrow$ cis isomerization is accompanied by a reversible 100 -fold change in the film conductance. In this way, the device can be set in a high- or low-resistance state by light irradiation at different wavelengths. This result paves the way towards the potential use of poly(metha)acrylate/azobenzene polymer films as active layer for optical input/electrical output memory elements.

\section{INTRODUCTION}

Polymer surfaces and interfaces can be designed by chemical programing in order to be capable of responding to external stimuli in a controlled and predictable manner. ${ }^{1,2}$ The incorporation of well-defined functional groups in the polymer main- or side-chain enables the generation of smart/dynamic soft materials, by offering additional remote controls (e.g. light, mechanical pressure, $\mathrm{pH}$, electric/magnetic fields, etc) to modulate their properties. Among various stimuli, light is a perfect choice since it is everlasting, it is clean as it leaves no side products, it features high spatio-temporal resolution and it is non-invasive on a wide range of wavelengths. The possibility to tune their wavelength and intensity guarantees a wealth of solutions when the photochromic systems are intelligently designed. Among various multifunctional polymers, poly(metha)acrylate/azobenzene polymers $(\mathrm{P}(\mathrm{MM}) \mathrm{A}-\mathrm{AZO})$ are well-established thermotropic photo-responsive polymers composed by photoswitchable azobenzene (AZO) side-groups bonded to the backbone of polymethacrylate (PMA) or polyacrylate (PA). ${ }^{3,4}$ While PMA and PA are widely-used dielectric polymers for electronic applications, ${ }^{5,6} \mathrm{AZO}$ derivatives are a class of diazene capable of undergoing reversible photochemical isomerization between a trans and a cis state. $^{7,8}$ When the two systems are covalently linked to the macromolecular side-chain the dielectric properties of PMA and PA are combined to the optical responsive nature of AZO 
moieties, and the whole macromolecule is affected by cis/trans photoisomerization of the AZO groups. ${ }^{9,10}$ If the polymer is in its liquid crystal phase, the AZO photochemical isomerization is accompanied by a profound structural and morphological rearrangement. ${ }^{11,12}$ Taking advantage of this unique feature, $\mathrm{P}(\mathrm{M}) \mathrm{A}-\mathrm{AZO}$ have been successfully employed as active materials in optical memories, in which optical information could be stored into $\mathrm{P}(\mathrm{M}) \mathrm{A}-\mathrm{AZO}$ thin films by laser or polarized light induced modification of surface gratings or birefringence. ${ }^{13-15}$ The information could be read with various optical techniques, such as birefringence, ${ }^{13}$ chiroptical devices, ${ }^{16,17}$ anisotropy analysis ${ }^{18}$ and SHIM microscopy ${ }^{19}$ resulting in optical-input, opticaloutput memories.

These approaches indicate that AZO polymers are emerging as promising active layers for memories. They can be considered as transducers converting photons into mechanical energy in the form of conformational change. ${ }^{20}$ However, in order to integrate these elements in binary electronic circuits there is a need to further transform the conformational change into an electrical output signal. ${ }^{21,22}$ The replacement of electrical stimuli with light inputs would enable to improve electrical performances offering orthogonal input-output signals. ${ }^{23}$ It also would offer interesting perspectives towards novel multifunctional devices. ${ }^{24}$ Such a goal has been pursued by employing photochromic molecules in organic/hybrid electronic devices. ${ }^{25}$ On this regard, azobenzenes molecules have been embedded in molecular junctions for memory applications. ${ }^{8,26}$ Moreover, AZO-polymers have been explored as active element in electrical devices by taking advantage of the photo-induced isomerization to modulate charge trapping in two terminal devices. ${ }^{27,28}$ However, all these previously reported approaches do not exploit the light responsivity nature of the AZO unit to modulate electrical properties of a devices but they rather 
exploit electrical inputs. Moreover, they exhibit very low current flowing through the device due to the insulating nature of AZO-polymers.

In this work, we show that light-induced morphology modifications in the $\mathrm{P}(\mathrm{M}) \mathrm{A}-\mathrm{AZO}$ polymer can be directly detected electrically via a difference in the film conductance. In particular, we have made use of a simple two-terminal vertical device geometry in which a very thin film of AZO-polymer is sandwiched between a solid metallic Au electrode and a top contact made of a soft drop of Gallium Indium (E-GaIn) eutectic. This innovative approach makes it possible to increase the current flowing through to the device and most importantly, to record the electrical memory information directly with light stimuli. We employed two AZO-polymers (Figure 1a), i.e. poly [4-pentiloxy-3'methyl- 4'-(6-acryloylo xyhexyloxy) azobenzene] called PA-AZO1 and poly[4-ethoxy-4'-(6-methacryloyloxyhexyloxy)azobenzene] named PMA-AZO2 which at room temperature (RT) are in a liquid crystalline $(\mathrm{LC})^{29}$ and in a glass phase, ${ }^{30,31}$ respectively. We show that while the electrical characteristics of the glass-phase PMA-AZO2 $\left(\mathrm{T}_{\mathrm{g}}=76{ }^{\circ} \mathrm{C}\right)$ thin films are not affected by the AZO photochemical isomerization, the thin films of the LC PAAZO1 $\left(\mathrm{T}_{\mathrm{g}}=19^{\circ} \mathrm{C}\right)$ are characterized by two distinct electrically-readable conductive states which can be reversibly addressed by non-polarized light irradiation at different wavelengths. In this way, PA-AZO1 thin-films behave as active layer in an optical input/electrical output memory element, in which the data can be electrically read, and optically written/erased.

\section{RESULTS AND DISCUSSION}

The optical properties PA-AZO1 and PMA-AZO2 were investigated in spin-coated thin films. The UV-vis spectra of pristine films are displayed in Figure 1b,c (black curves). They exhibit an 
absorption band at around $360 \mathrm{~nm}$, typical of $\pi-\pi^{*}$ transition of AZO moieties in trans configuration with two additional shoulders at 340 and $380 \mathrm{~nm}$, which can be ascribed respectively to the formation of $\mathrm{H}$ - (blue-shift) and J-like (red-shift) aggregates imposed by the structural constraints of the macromolecules in the solid state ${ }^{32}$. The relatively high absorbance of the transition at $340 \mathrm{~nm}$ indicates a high concentration of $\mathrm{H}$ aggregates. For both polymers, a reduction in the intensity of such bands was observed after irradiation with non-polarized UV light (365 nm, $5 \mathrm{~min}$ ) whereas the intensity of the $\mathrm{n}-\pi^{*}$ band at $450 \mathrm{~nm}$, typical of the cis form, slightly increased (Figure 1b,c the red line). Upon irradiation with non-polarized visible light (455 nm), both polymer films show a partial recovery to initial absorbance features (Figure 1b,c magenta $v$ black spectrum) and the absence of classic isosbestic point. ${ }^{33}$ The intensity of the recovered band depends on the irradiation time (see Figure 1b,c, magenta vs green line):

a)

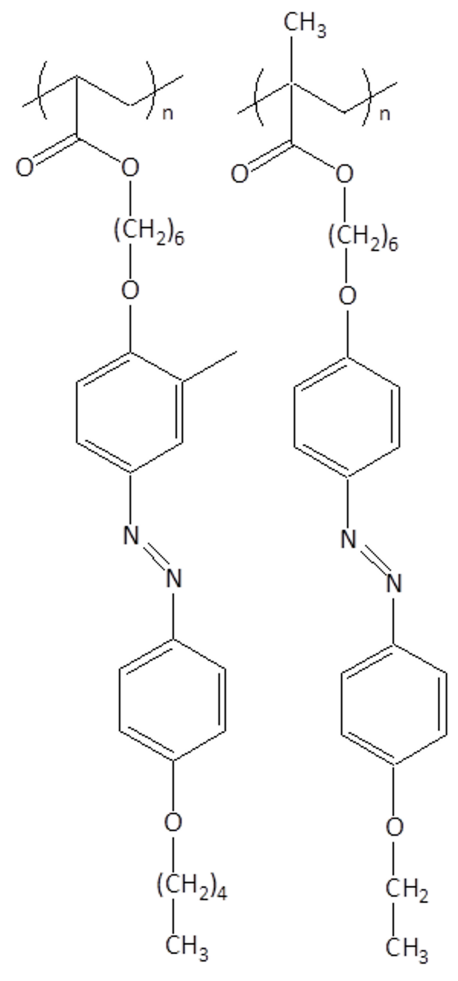

PA-AZO1

PMA-AZO2

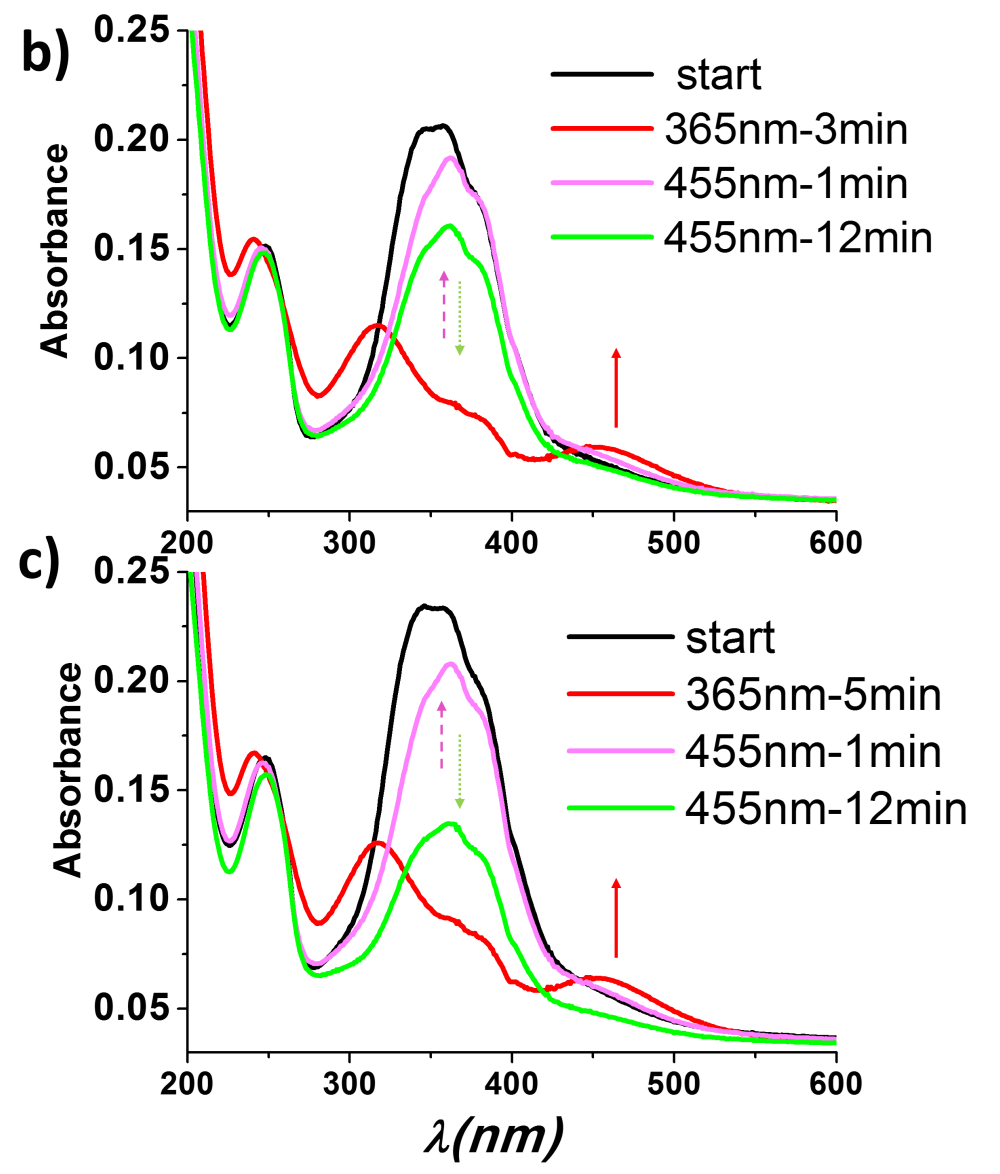


Figure 1 a) chemical structure of PA-AZO1 and PMA-AZO2. b) PA-AZO1 and c) PMA-AZO2 changes in absorption spectra of a thin film of by irradiation with $365 \mathrm{~nm}$ and $455 \mathrm{~nm}$. Initial trans-form (black line), is irradiated for 3-5 minutes with $365 \mathrm{~nm}$ centered light to obtain cisform (red line). Successive recovery of the trans-form by irradiation with $455 \mathrm{~nm}$ : after 1 minutes is reported in magenta. Continuous irradiation with $455 \mathrm{~nm}$ light produces a decrease in maximum peak absorbance spectra (green).

Prolonged irradiation induces a decrease of the absorbance band. As previously reported, the change in the shape of the absorbance bands during the irradiation time can be ascribed to the presence of out-of-plane aggregates. ${ }^{34}$ In particular, the irradiation at $455 \mathrm{~nm}$ favors J-aggregates formation (centered at $380 \mathrm{~nm}$ ) rather than $\mathrm{H}$-aggregates (band at $340 \mathrm{~nm}$ ).

This is supported by X-ray Diffraction measurements revealing a $4.48 \AA$ periodicity along the film plane of the trans-form of PA-AZO1 which can be attributed to the presence of $\mathrm{H}$ aggregation in the liquid crystal film (see Figure S1) The coherence length of these aggregates is $\sim 2.4 \mathrm{~nm}$ indicating a short-range order nature of the liquid crystalline aggregates.

This finding indicates that in PA-AZO1 films the photochromic meso-groups interact among each other. It is well known that photo-responsive mechanical properties of AZO polymers depend on the interactions between the AZO moieties. These interactions can determine the emergence of a certain degree of organization within the materials (e.g. characterized by a nematic phase, in the specific case), leading to the generation of different structures upon irradiation. ${ }^{35}$ Most recent theoretical approaches suggest that the reorientation of the azo-group is changed when they are exposed to light linearly polarized perpendicularly, dragging the polymer backbone, ultimately leading to morphological changes in the materials. ${ }^{36}$ It has also been 
verified that the irradiation intensity plays a role in the photo-isomerization and consequently in the morphological rearrangement. ${ }^{37}$ Because of these reasons, we decided to use always the same light intensity and the same irradiation time when characterizing the electrical devices. Also, for the sake of simplicity and easier applicability, non-polarized light has been employed.

In order to study how the morphology of the spin-coated polymers is affected by the trans-to-cis photochemical isomerization, we have performed Atomic Force Microscopy (AFM) measurements on both PMA-AZO2 and PA-AZO1 before and after irradiation with light at 365 nm (Figure 2). The polymers in the as-spin-coated films are in their trans form. The as-spincoated PA-AZO1 film is characterized by the presence of nanostructures protruding out of the surface and appearing as nano-hills (Figure 2a).
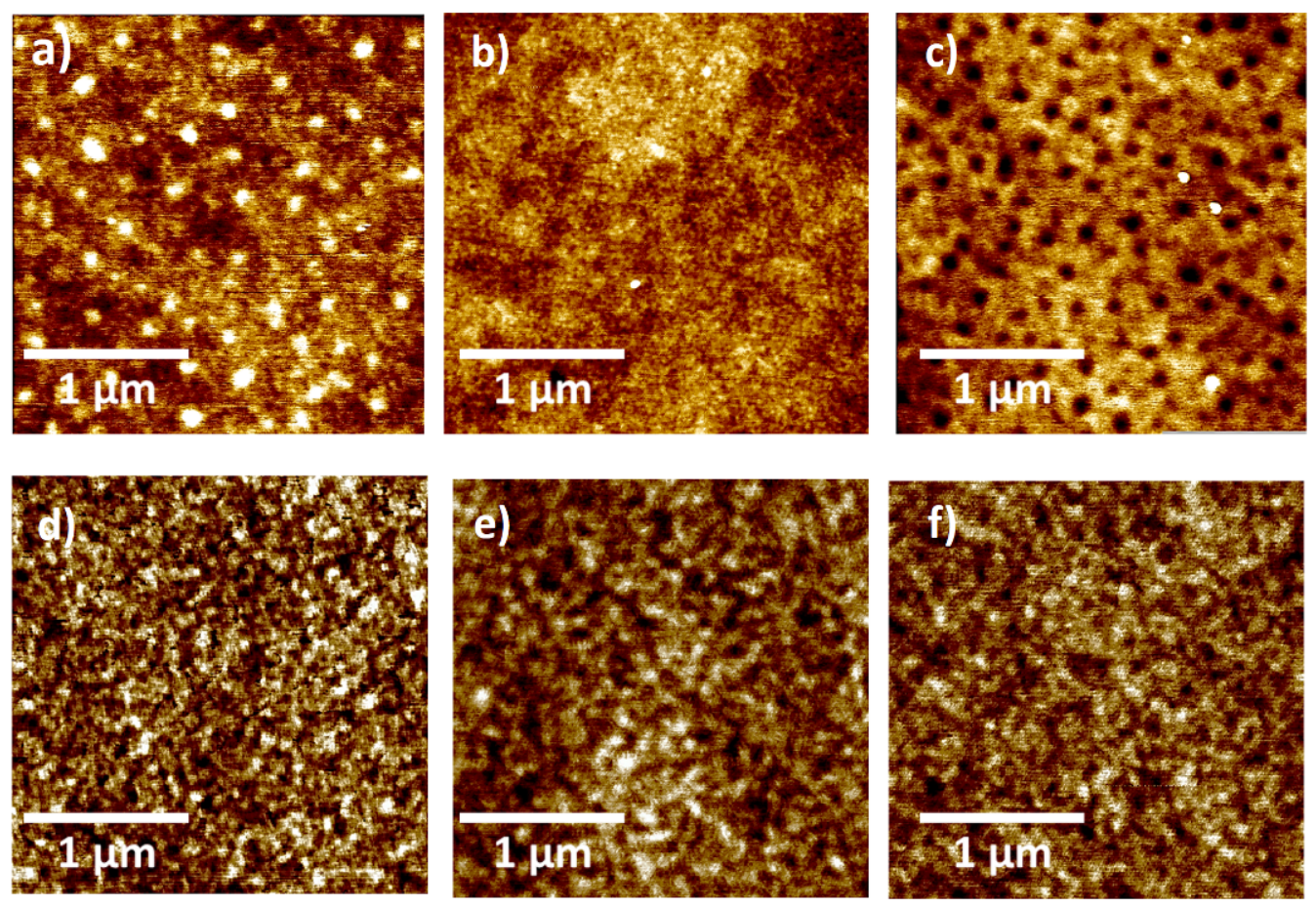

Figure 2 a) AFM topographical images of PA-AZO1 film before irradiation, b) after $5 \mathrm{~min}$ irradiation at $\lambda=365 \mathrm{~nm}$, and c) after 15 min irradiation at $\lambda=365 \mathrm{~nm}$. d) AFM topographical 
images of PMA-AZO2 film before irradiation, e) after 5 min irradiation at $\lambda=365 \mathrm{~nm}$, and f) after 15 min irradiation at $\lambda=365 \mathrm{~nm}$. Z-scales $=3 \mathrm{~nm}$..

The coherence length of these aggregates is $\sim 2.4 \mathrm{~nm}$ indicating a short-range order nature of the liquid crystalline aggregates. To better analyze how the morphology of the so-deposited polymers is affected by the trans-to-cis photochemical isomerization, we have performed Atomic Force Microscopy (AFM) measurements on both PMA-AZO2 and PA-AZO1 before and after irradiation with light at $365 \mathrm{~nm}$ (Figure 2). The polymers in the as-spin-coated films are in their trans form. The as-spin-coated PA-AZO1 film is characterized by the presence of nanostructures protruding out of the surface and appearing as nano-hills (Figure 2a). These features are typically $2 \mathrm{~nm}$ tall, about 60-80 nm wide, and are randomly distributed over the whole surface. Such a morphology, on a length scale of $10 * 10 \mu \mathrm{m}^{2}$ exhibits a root-mean-square roughness $\mathrm{R}_{\mathrm{RMS}}$ $=0.51 \mathrm{~nm}$. In this case, irradiation with UV-light determines a profound surface rearrangement (see, Figure 2a vs 2b). In particular, 5 min irradiation with $365 \mathrm{~nm}$ light led to a disappearance of the characteristic nano-hills of the pristine PA-AZO1 film, and the surface becomes smoother as evidenced by the decrease in $\mathrm{R}_{\mathrm{RMS}}$ from 0.51 to $0.40 \mathrm{~nm}$. A 15-minute UV light exposure led to a completely different morphology, characterized by 2-nm-deep and about 70-nm wide dips on the sample surface, as highlighted in Figure 3c. Multiple irradiation cycles have been performed to promote the reversible isomerization from the trans to the cis form and vice versa. They revealed consecutive changes of surface morphology upon irradiation between the different surface morphologies (Figure S3). Moreover, the two different states are stable for over one week (Figure S8). Importantly, the 2-nm deep dimples produce a local change in the effective film thickness; however, since the reorganization is confined at the top surface, the overall thickness of the films does not change significantly upon irradiation. (see Figure S4). We 
highlight that films of different thicknesses are characterized by very similar morphology, as shown in the SI.

Conversely, the pristine PMA-AZO2 film is characterized by a very smooth surface $\left(\mathrm{R}_{\mathrm{RMS}}=\right.$ $0.35 \mathrm{~nm}$, Figure 2a). Interestingly, Figure 2b,c shows that the film morphology is unchanged when the surface is irradiated with a LED at $365 \mathrm{~nm}$. The obtained results are in good agreement with the data reported in literature related to the formation of nano-structures on the surface of $\mathrm{AZO} / \mathrm{P}(\mathrm{M}) \mathrm{A}$ polymers, ${ }^{12}$ as well as the formation of an intermediate phase of reorganization. ${ }^{38,39}$ In particular, the morphological rearrangement only occurs if the LC polymer is not in its glass phase at the operating temperature condition. With regard to our systems, at RT only PA-AZO1 is in its nematic LC phase $\left(\mathrm{T} g=19{ }^{\circ} \mathrm{C}\right)$, explaining why it undergoes a dramatic morphological reorganization when irradiated with UV-Vis light. ${ }^{40,41}$ Moreover has been reported by Angiolini et al. in the PA-AZO1 the isomerization from trans to cis generates a contraction of the material.

On the other hand, PMA-AZO2 has a $\mathrm{T}_{\mathrm{g}}=76^{\circ} \mathrm{C}$. Such a higher $\mathrm{T}_{\mathrm{g}}$ is determined by the change to methyl-acrylate of the backbone chain. Therefore the polymer chains are frozen in their position after the deposition at room temperature, ${ }^{30}$ and this likely does not allow their sidechains to undergo reorganization, hindering the formation of surface nano-hills and nano-dips. ${ }^{41}$

To provide evidence of the important role played by temperature on the film morphology, we investigated by AFM the effect of the light irradiation on PA-AZO1 film at $\mathrm{T}<\mathrm{Tg}$ in order to keep it in its glass phase (see Figure S5). Our optical results show that AZO-groups inside the polymer film undergo photochemical isomerization even in the glass state (PMMA-AZO2, Figure 1c), yet AFM characterization reveals that the polymer is too rigid to undergo morphological reorganization on the large scale. Conversely, when the polymer enters its LC 
phase upon increase of the operating temperature up to $25 \mathrm{C}$ degrees, we can observe the morphological reorganization leading to the formation of surface nano-features. This suggests that polymeric chains are curled around rod-like aggregated structures ( $\mathrm{H}$ and $\mathrm{J}$ aggregates) composed of the AZO side-groups clenched together and immersed in a matrix of amorphous $\mathrm{P}(\mathrm{M}) \mathrm{A}$ main-chains as for similar polymers. ${ }^{42}$ Switching from trans to cis can therefore trigger a conformational structural re-organization within these areas. ${ }^{43},{ }^{44}$

In order to characterize the electrical properties of the azoaromatic polymer films, we have employed a two-terminal vertical device geometry based on an Eutectic Gallium/Indium (EGaIn) drop as top electrode. E-GaIn is an ideal material for the measurement of tunneling resistance in self-assembled monolayers, ${ }^{45,46}$ as well across polymeric thin film. ${ }^{47}$ Because of its non-newtonian behavior, it can be easily shaped into sharp tips which are suitable to make conformal non-damaging contacts with the substrate and therefore it allows to achieve reproducible non-destructive current density-voltage (J-V) measurements. ${ }^{48}$ Following the same procedure reported for previous analysis, we prepared PA-AZO1 and PMA-AZO2 $8 \mathrm{~nm}$ thick films to study the current flowing through them using this vertical junction geometry. The results presented here are an average of at least 500 voltage sweeps for each ON or OFF state. (i.e. trans or cis configuration) recorded in different days in a not conditioned room with temperatures ranging between $22{ }^{\circ} \mathrm{C}$ and $26^{\circ} \mathrm{C}$. An example of a curve obtained in the linear scale can be found in the Figure S9. We always used the non-polarized light sources with the same irradiation power for all the devices $\left(52 \mathrm{mWcm}^{-2}\right.$ for $365 \mathrm{~nm}$ LED and $0.45 \mathrm{mWcm}^{-2}$ for $455 \mathrm{~nm}$ LED). A schematic illustration of E-GaIn drop in contact with the upper surface of the polymer is shown in Figure 3a. PA-AZO1 films have been spin-coated on a gold electrode evaporated over a quartz slide, with a thin chromium layer in-between to improve Au adhesion. 
As reported in Figure 3b, the PA-AZO1 film exhibits an increase of conductance of almost 2 orders of magnitude after $15 \mathrm{~min}$ of in-situ irradiation with $365 \mathrm{~nm}$ UV light. As expected by the optical and morphological characterization, the photo-isomerization is reversible, and also the current density in the PA-AZO1 can be switched back to the original value (green line) after retro-isomerization to the trans azobenzene form irradiating the device with light at $455 \mathrm{~nm}$ (Figure 3b). Therefore, we can regard the PMA-AZO1 vertical junction as a bistable memory element with a high resistive OFF state in the trans form and a low resistive ON state in the cis form. In such memory, the data are "written" by irradiation at $365 \mathrm{~nm}$, "read" electrically and "erased" by irradiation at $455 \mathrm{~nm}$. Also we tested the morphological reorganization observed in Figure 2b with an intermediate state in which no nanostructures are present (irradiation with UV-light for 5 minutes). The film conductance associated with this in-between state is halfway the ON and OFF states (Figure S10). The ON/OFF ratio in a memory is given by the ratio between the conductance of the high conductive state and the low conductive state, and it reaches 95 in this case.

PMA-AZO2, which is in its glass phase, has a completely different behavior. Initially, the current through the pristine trans PMA-AZO2 film is one order of magnitude lower than that in PA-AZO1 (compare Figure 3b,c). Then, the current does not change significantly upon irradiation at $\lambda=365 \mathrm{~nm}$ and at $\lambda=455 \mathrm{~nm}$, as displayed in Figure 3c. We ascribe the different electrical performances to the different morphological behavior of the AZO-polymer films when irradiated with UV light at RT. As discussed above, when PA-AZO1 thin-films are in their LCstate they can undergo structural reorganization as a result of light irradiation. Conversely, the 
morphology of glassy PMA-AZO2 is hardly affected by the irradiation, and it is consequently characterized by only one resistance state.
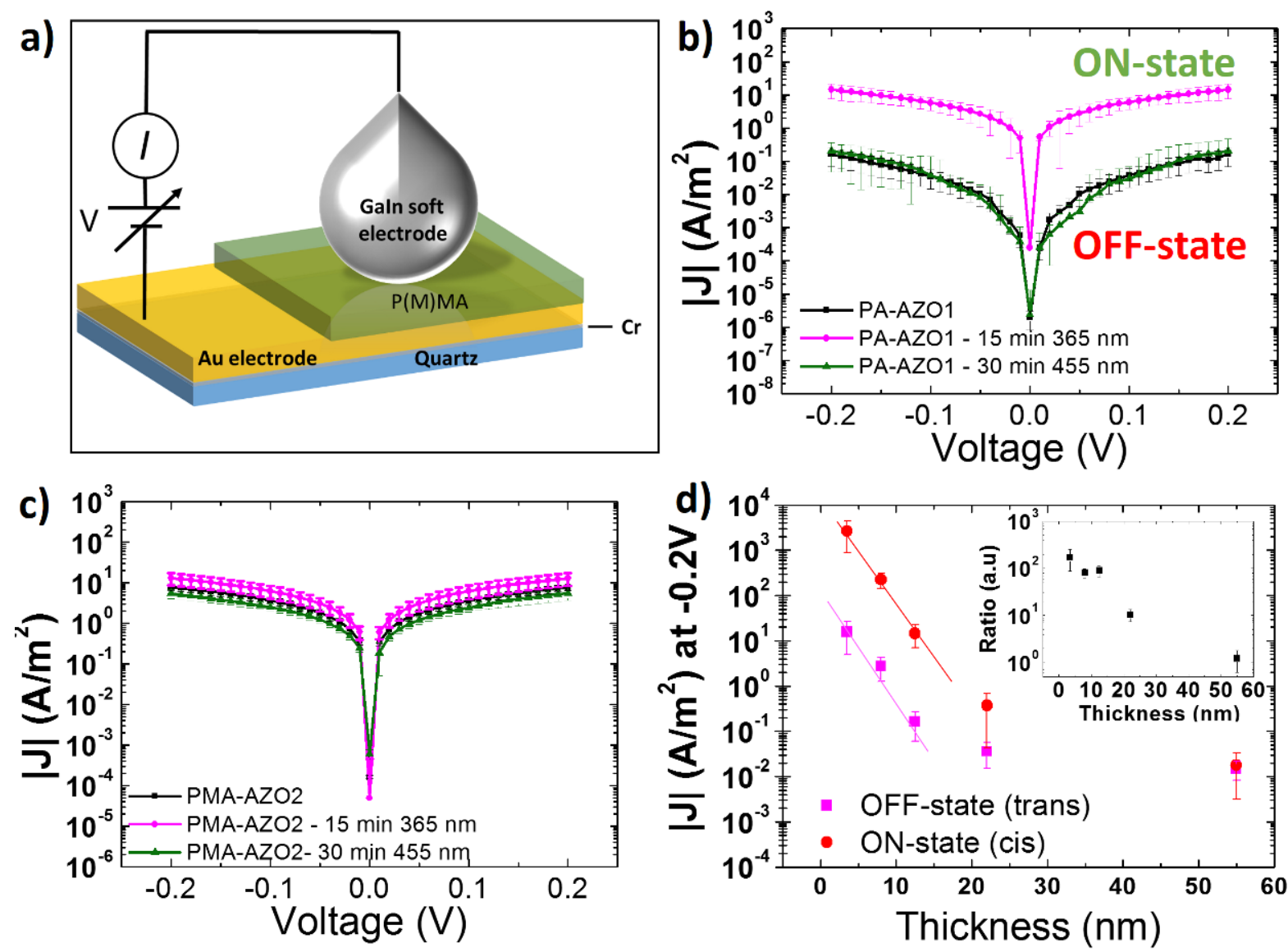

Figure 3 a) Schematic representation of vertical geometry employed, showing top E-GaIn electrode contacted on top of azo-aromatic polymeric thin film. An Au bottom electrode on quartz is grounded, while the potential is applied through top (soft) electrode for b) PA-AZO1 and c) PMA-AZO2. In black are reported initial measurements, in violet after $15 \mathrm{~min}$ at $\lambda=365$ $\mathrm{nm}$ irradiation and in green after consequently $30 \mathrm{~min}$ at $\lambda=455 \mathrm{~nm}$ irradiation. d) Summary plot of J value in OFF-state (trans magenta) and ON state (cis red) at $-0.2 \mathrm{~V}$ plotted vs. film thickness of PA-AZO1. Also the single exponential fitting of the first three points is represented. The inset displays the ratio $\mathrm{I}_{\mathrm{ON}} / \mathrm{I}_{\mathrm{OFF}}$ as a function of the thickness. 
To optimize the $\mathrm{I}_{\mathrm{ON}} / \mathrm{I}_{\mathrm{OFF}}$ ratio, we investigated PA-AZO1 films with different thicknesses: 3,8 , 12, 22 and $55 \mathrm{~nm}$ by using the same vertical junction configuration. It is worth pointing out that the same surface morphology has been observed for all the studied film thickness (details in SI Figure S12). The experimental results are summarized in Figure 3d. The latter shows the current density values at $-0.2 \mathrm{~V}$ for different thickness (Figure S7). Interestingly, the current decreases exponentially with the polymer nominal thickness in the range between 0 and $12 \mathrm{~nm}$, for both films with the azo moieties in their trans and cis state. This behavior is typically observed for electron transport in tunneling regime. ${ }^{49}$ In reality, $10 \mathrm{~nm}$-thick films should not allow charge tunneling, but the effective film thickness might be significantly lower than the nominal one due to thinner pathways across the film. Indeed, the surface of the bottom gold electrode has a greater roughness compared to the one observed for the polymer deposited on an ultraflat substrate. The Au electrode has a $\mathrm{R}_{\mathrm{RMS}}$ of $0.62 \mathrm{~nm}$, for surfaces like the one displayed in the AFM image in Figure S11. As a result, the thickness of the polymer is not constant through the junction area, but it depends locally on the roughness of bottom gold surface. This concept is schematically shown in Figure 4, in which a schematic side-view of our vertical junction is drawn employing representative AFM profiles actually measured for the gold bottom contact and the PA-AZO-1 in the cis and trans state. The profiles are extracted from real AFM images and are drawn using the same vertical scale. In a tunneling framework, the exponential dependence of the current on the barrier thickness implies that the current does not flow homogeneously throughout the junction area, but it is rather dominated by those hot spots in which the films is thinner. ${ }^{50}$ Therefore, the effective thickness of our junctions might be significantly lower than the nominal thickness. This effect is often observed for solid-state tunnel junctions ${ }^{51}$ and is particularly significant in our case, since the thickness of the polymer varies significantly due to 
the high roughness of the bottom gold. Based on the Au roughness, we argue that the thickness of the thinnest regions of the polymer are thin enough to permit electron tunneling even in the case of the nominally $12 \mathrm{~nm}$-thick polymer film.

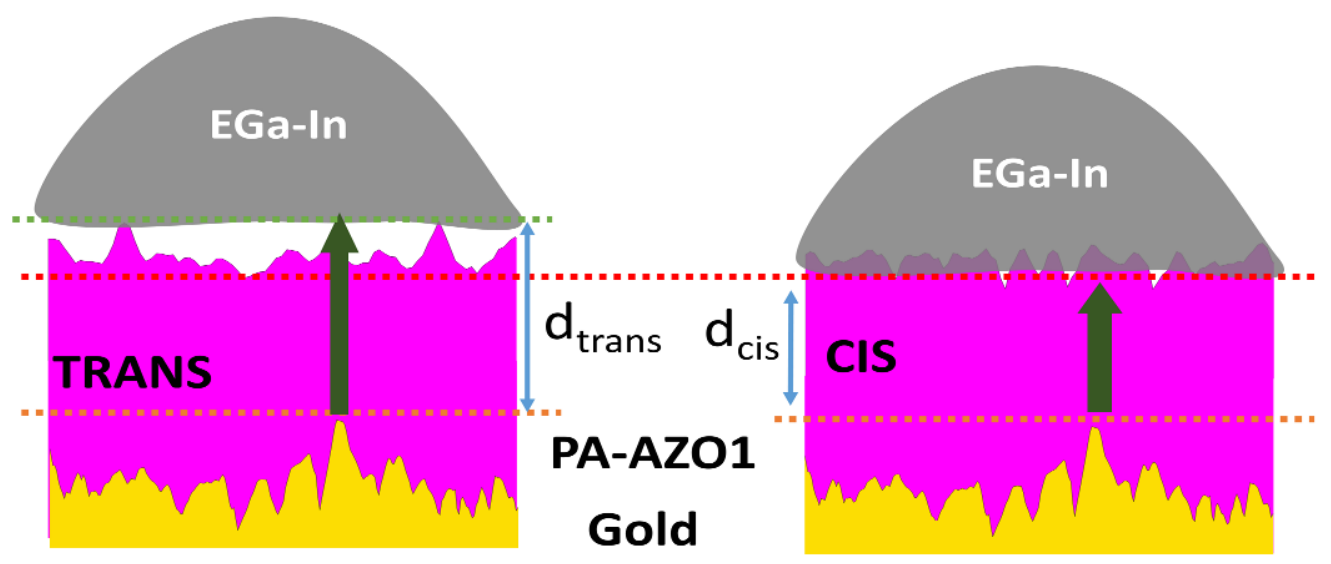

Figure 4 Schematic representation of morphology modification induced by light. Real representative profiles from AFM are shown for both gold electrode, PA-AZO1 in its a) trans and b) cis form. The distance between the two electrodes is reported as $d_{\text {trans }}$ and $d_{\text {cis. }}$. The green arrow indicates the effective distance between the electrodes in the two different states.

For samples above $12 \mathrm{~nm}$, the exponential decrease of the current with the film thickness is no longer valid. We conclude that in this case even in those spots where the film thickness is minimum, tunneling is prohibited because the films are too thick. Other charge transport mechanism will take place in this range.

Figure 3d also shows the change in the current with irradiation for films with different thicknesses. The highest relative ratio between the two conductive states is about 170 and it is measured for the thinnest film (nominally $3 \mathrm{~nm}$ thick), while there is no conductivity change for the thickest film (nominally $50 \mathrm{~nm}$ thick). This change in conductivity could be caused by a change in the effective contact area or by a change in the film thickness. The effect of the two 
contributions is different, as detailed in the SI, and we conclude that the thickness change is actually responsible for the change in conductance.

While the cartoon in Figure 4 does not take into account the roughness of the $\mathrm{Ga}_{2} \mathrm{O}_{3}$, albeit important in the determination of the effective contact area, ${ }^{52}$ it is reasonable to assume that the thickness of the polymer is, on average, higher in the trans state. Based on the AFM characterization, we estimate a two-nanometer difference in effective thickness for the cis and trans state for all the thicknesses explored in this study. For this reason, highest current is expected in the cis case, in good agreement with what we measure. Moreover, the highest change in current is measured for the thinnest film, because the relative variation of the thickness is highest in this case. For thick films (>20 nm), where tunneling is not the main charge transport mechanism, the photo-induced thickness variation becomes negligible, and accordingly we do not measure difference in the resistance of the trans and cis states. We highlight that the current in the trans state of the nominally 3-nm-thick film has the same order of magnitude of the current flowing in the cis state of the nominally 12 -nm-thick film. This observation suggests that the effective thickness for the two films is similar, further confirming that the nominal thickness is significantly higher than the effective one.

In light of these findings, we can consider the whole sample like a light programmable bi-stable memory. Contacting the film with a top soft junction in any point of the surface enables an easy scanning of the light-programmed memory state.

CONCLUSION 
In summary, we have realized a polymeric thin-film based light-driven multifunctional memory element, in which conductance is programmed via light irradiation at specific wavelengths and the state of the system is read out electrically. This was made possible as a result of the strict correlation between light irradiation and surface's nano-structures morphological modification of PA-AZO1 polymer, which assembles in its liquid crystalline phase into aggregates. The whole PA-AZO1 film is the active switchable layer of the memristor, meaning that a punctual and reproducible read of the memory state can be done using a top soft junction. Our result, based on the use of optical input/electrical output memories is easily applicable to different optically responsive polymer and oligomer-based materials, and it paves the way towards optical multiresponsive integrated memory devices.

\section{ASSOCIATED CONTENT}

Supporting Information. Chemical synthesis and polymer characterizations, AFM observation of multiple cycles, X-RAY measurements, electrical characterization of all the different samples. This material is available free of charge via the Internet at http://pubs.acs.org.

\section{AUTHOR INFORMATION}

\section{Corresponding Author}

*Paolo Samorì samori@unistra.fr

\section{Author Contributions}

All authors have given approval to the final version of the manuscript.

\section{ACKNOWLEDGMENT}


We would like to thank Dr. Oliver Fenwick and Mr. Marco Squillaci for useful discussions. This work was financially supported by the EC through the Marie Sklodowska-Curie ITN project iSwitch (GA-642196) and the ERC project SUPRAFUNCTION (GA-257305), the Agence Nationale de la Recherche through the LabEx CSC (ANR-10-LABX-0026_CSC) and the International Center for Frontier Research in Chemistry (icFRC). SB thanks the EC Marie Curie - IEF fellowship GALACTIC (PIEF-GA-2013-628563).

\section{REFERENCES}

1. Stuart, M. A. C.; Huck, W. T. S.; Genzer, J.; Muller, M.; Ober, C.; Stamm, M.; Sukhorukov, G. B.; Szleifer, I.; Tsukruk, V. V.; Urban, M.; Winnik, F.; Zauscher, S.; Luzinov, I.; Minko, S., Emerging Applications of Stimuli-Responsive Polymer Materials. Nat Mater 2010, 9, 101-113.

2. Luzinov, I.; Minko, S.; Tsukruk, V. V., Adaptive and Responsive Surfaces through Controlled Reorganization of Interfacial Polymer Layers. Prog Polym Sci 2004, 29, 635-698.

3. Natansohn, A.; Rochon, P., Photoinduced Motions in Azo-Containing Polymers. Chem. Rev. 2002, 102, 4139-4176.

4. Plain, J.; Wiederrecht, G. P.; Gray, S. K.; Royer, P.; Bachelot, R., Multiscale Optical Imaging of Complex Fields Based on the Use of Azobenzene Nanomotors. J. Phys. Chem. Lett. 2013, 4, 2124-2132.

5. Hayashida, K., Dielectric Properties of Polymethacrylate-Grafted Carbon Nanotube Composites. RSC Adv. 2013, 3, 221-227.

6. Wunsche, J.; Tarabella, G.; Bertolazzi, S.; Bocoum, M.; Coppede, N.; Barba, L.; Arrighetti, G.; Lutterotti, L.; Iannotta, S.; Cicoira, F.; Santato, C., The Correlation between Gate Dielectric, Film Growth, and Charge Transport in Organic Thin Film Transistors: The Case of Vacuum-Sublimed Tetracene Thin Films. J. Mater. Chem. C 2013, 1, 967-976.

7. Bandara, H. M. D.; Burdette, S. C., Photoisomerization in Different Classes of Azobenzene. Chem. Soc. Rev. 2012, 41, 1809-1825. 
8. Osella, S.; Samorì, P.; Cornil, J., Photoswitching Azobenzene Derivatives in Single Molecule Junctions: A Theoretical Insight into the I/V Characteristics. J. Phys. Chem. C 2014, $118,18721-18729$.

9. $\quad$ Barrett, C. J.; Mamiya, J.-i.; Yager, K. G.; Ikeda, T., Photo-Mechanical Effects in Azobenzene-Containing Soft Materials. Soft Matter 2007, 3, 1249-1261.

10. Ikeda, T.; Ube, T., Photomobile Polymer Materials: From Nano to Macro. Mater. Today 2011, 14, 480-487.

11. Yadavalli, N. S.; Korolkov, D.; Moulin, J.-F.; Krutyeva, M.; Santer, S., Probing OptoMechanical Stresses within Azobenzene-Containing Photosensitive Polymer Films by a Thin Metal Film Placed Above. ACS Appl. Mater. Interfaces 2014, 6, 11333-11340.

12. Zhao, Y.; He, J., Azobenzene-Containing Block Copolymers: The Interplay of Light and Morphology Enables New Functions. Soft Matter 2009, 5, 2686-2693.

13. Ramanujam, P. S.; Hvilsted, S.; Ujhelyi, F.; Koppa, P.; Lörincz, E.; Erdei, G.; Szarvas, G., Physics and Technology of Optical Storage in Polymer Thin Films. Synth. Met. 2001, 124, 145-150.

14. Ishow, E.; Lebon, B.; He, Y.; Wang, X.; Bouteiller, L.; Galmiche, L.; Nakatani, K., Structural and Photoisomerization Cross Studies of Polar Photochromic Monomeric Glasses Forming Surface Relief Gratings. Chem. Mater. 2006, 18, 1261-1267.

15. Yadavalli, N. S.; Saphiannikova, M.; Santer, S., Photosensitive Response of Azobenzene Containing Films Towards Pure Intensity or Polarization Interference Patterns. Appl. Phys. Lett. 2014, 105, 051601-051605.

16. Angiolini, L.; Bozio, R.; Giorgini, L.; Pedron, D.; Turco, G.; Dauru, A., Photomodulation of the Chiroptical Properties of New Chiral Methacrylic Polymers with Side Chain Azobenzene Moieties. Chem. Eur. J. 2002, 8, 4241-4247.

17. Lu, J.; Jiang, G.; Zhang, Z.; Zhang, W.; Yang, Y.; Wang, Y.; Zhou, N.; Zhu, X., A Cyclic Azobenzenophane-Based Smart Polymer for Chiroptical Switches. Polym. Chem. 2015, 6, 81448149 .

18. Lee, M.-J.; Jung, D.-H.; Han, Y.-K., Photo-Responsive Polymers and Their Applications to Optical Memory. Mol. Cryst. Liq. Cryst. 2006, 444, 41-50.

19. Spiridon, M. C.; Iliopoulos, K.; Jerca, F. A.; Jerca, V. V.; Vuluga, D. M.; Vasilescu, D. S.; Gindre, D.; Sahraoui, B., Novel Pendant Azobenzene/Polymer Systems for Second Harmonic Generation and Optical Data Storage. Dyes Pigm. 2015, 114, 24-32.

20. Koerner, H.; White, T. J.; Tabiryan, N. V.; Bunning, T. J.; Vaia, R. A., Photogenerating Work from Polymers. Mater. Today 2008, 11, 34-42. 
21. Sun, C.; Wade, M. T.; Lee, Y.; Orcutt, J. S.; Alloatti, L.; Georgas, M. S.; Waterman, A. S.; Shainline, J. M.; Avizienis, R. R.; Lin, S.; Moss, B. R.; Kumar, R.; Pavanello, F.; Atabaki, A. H.; Cook, H. M.; Ou, A. J.; Leu, J. C.; Chen, Y.-H.; Asanović, K.; Ram, R. J.; Popović, M. A.; Stojanović, V. M., Single-Chip Microprocessor That Communicates Directly Using Light. Nature 2015, 528, 534-538.

22. Raymo, F. M., Digital Processing and Communication with Molecular Switches. Adv. Mater. 2002, 14, 401-414.

23. Ríos, C.; Stegmaier, M.; Hosseini, P.; Wang, D.; Scherer, T.; Wright, C. D.; Bhaskaran, H.; Pernice, W. H. P., Integrated All-Photonic Non-Volatile Multi-Level Memory. Nat Photon 2015, 9, 725-732.

24. Andréasson, J.; Pischel, U.; Straight, S. D.; Moore, T. A.; Moore, A. L.; Gust, D., AllPhotonic Multifunctional Molecular Logic Device. J. Am. Chem. Soc. 2011, 133, 11641-11648.

25. Orgiu, E.; Samorì, P., 25th Anniversary Article: Organic Electronics Marries Photochromism: Generation of Multifunctional Interfaces, Materials, and Devices. Adv. Mater. 2014, 26, 1827-1845.

26. Min, M.; Seo, S.; Lee, S. M.; Lee, H., Voltage-Controlled Nonvolatile Molecular Memory of an Azobenzene Monolayer through Solution-Processed Reduced Graphene Oxide Contacts. Adv. Mater. 2013, 25, 7045-7050.

27. Attianese, D.; Petrosino, M.; Vacca, P.; Concilio, S.; Iannelli, P.; Rubino, A.; Bellone, S., Switching Device Based on a Thin Film of an Azo-Containing Polymer for Application in Memory Cells. Electron Device Letters, IEEE 2008, 29, 44-46.

28. Lim, S. L.; Li, N.-J.; Lu, J.-M.; Ling, Q.-D.; Zhu, C. X.; Kang, E.-T.; Neoh, K. G., Conductivity Switching and Electronic Memory Effect in Polymers with Pendant Azobenzene Chromophores. ACS Appl. Mater. Interfaces 2009, 1, 60-71.

29. Angeloni, A. S.; Caretti, D.; Laus, M.; Chiellini, E.; Galli, G., Mesomorphic Polyacrylates Containing Isomeric Methyl-Substituted Azobenzene Mesogens. J. Polym. Sci., Part A: Polym. Chem. 1991, 29, 1865-1873.

30. Angiolini, L.; Barberá, J.; Benelli, T.; Giorgini, L.; Paris, F.; Salatelli, E.; Tejedor Rosa, M., Photoinduced Supramolecular Chirality in Glassy Nematic Linear and Star Shaped Methacrylic Azopolymers Obtained by Atrp. e-Polymers 2009, 9, 553.

31. Angiolini, L.; Benelli, T.; Giorgini, L.; Paris, F.; Salatelli, E.; Fontana, M. P.; Camorani, P., Synthesis by Atrp and Effects of Molecular Weight on Photomechanical Properties of Liquid Crystalline Polymers Containing Side-Chain Azobenzene Chromophores. Eur. Polym. J. 2008, 44, 3231-3238.

32. Shimomura, M.; Kunitake, T., Fluorescence and Photoisomerization of AzobenzeneContaining Bilayer-Membranes. J. Am. Chem. Soc. 1987, 109, 5175-5183. 
33. Williams, I.; Williams, N., Advances in Physical Organic Chemistry. Elsevier Science: 2014.

34. Moritsugu, M.; Kim, S.-n.; Kubo, S.; Ogata, T.; Nonaka, T.; Sato, O.; Kurihara, S., Photoswitching Properties of Photonic Crystals Infiltrated with Polymer Liquid Crystals Having Azobenzene Side Chain Groups with Different Methylene Spacers. React. Funct. Polym. 2011, 71, 30-35.

35. Petrova, T.; Toshchevikov, V.; Saphiannikova, M., Light-Induced Deformation of Polymer Networks Containing Azobenzene Chromophores and Liquid Crystalline Mesogens. Soft Matter 2015, 11, 3412-3423.

36. Ilnytskyi, J. M.; Neher, D.; Saphiannikova, M., Opposite Photo-Induced Deformations in Azobenzene-Containing Polymers with Different Molecular Architecture: Molecular Dynamics Study. J. Chem. Phys 2011, 135, 044901.

37. Yadavalli, N. S.; König, T.; Santer, S., Selective Mass Transport of AzobenzeneContaining Photosensitive Films Towards or Away from the Light Intensity. Jnl. Soc. Info. Display 2015, 23, 154-162.

38. Barbera, J.; Giorgini, L.; Paris, F.; Salatelli, E.; Tejedor, R. M.; Angiolini, L., Supramolecular Chirality and Reversible Chiroptical Switching in New Chiral Liquid-Crystal Azopolymers. Chem. Eur. J. 2008, 14, 11209-11221.

39. Camorani, P.; Cristofolini, L.; Galli, G.; Fontana, M. P., Photoinduced Morphological Changes and Optical Writing in a Liquid Crystalline Polymer on the Micron and Sub-Micron Scale. Mol. Cryst. Liq. Cryst. 2002, 375, 175-184.

40. Zhao, Y., New Photoactive Polymer and Liquid-Crystal Materials. Pure Appl. Chem. 2004, 76, 1499-1508.

41. Matsuzawa, Y.; Tamaoki, N., Photoisomerization of Azobenzene Units Controls the Reversible Dispersion and Reorganization of Fibrous Self-Assembled Systems. J. Phys. Chem. B 2010, $114,1586-1590$.

42. $\quad$ Cai, Y.; Lu, J.; Zhou, F.; Zhou, X.; Zhou, N.; Zhang, Z.; Zhu, X., Cyclic Amphiphilic Random Copolymers Bearing Azobenzene Side Chains: Facile Synthesis and Topological Effects on Self-Assembly and Photoisomerization. Macromol. Rapid Commun. 2014, 35, 901907.

43. Bogdanov, A. V.; Vorobiev, A. K., Photo-Orientation of Azobenzene-Containing LiquidCrystalline Materials by Means of Domain Structure Rearrangement. J. Phys. Chem. B 2013, $117,13936-13945$.

44. Shimamura, A.; Priimagi, A.; Mamiya, J.-i.; Ikeda, T.; Yu, Y.; Barrett, C. J.; Shishido, A., Simultaneous Analysis of Optical and Mechanical Properties of Cross-Linked AzobenzeneContaining Liquid-Crystalline Polymer Films. ACS Appl. Mater. Interfaces 2011, 3, 4190-4196. 
45. Chiechi, R. C.; Weiss, E. A.; Dickey, M. D.; Whitesides, G. M., Eutectic GalliumIndium (Egain): A Moldable Liquid Metal for Electrical Characterization of Self-Assembled Monolayers. Angew. Chem. Int. Edit. 2008, 120, 148-150.

46. Masillamani, A. M.; Crivillers, N.; Orgiu, E.; Rotzler, J.; Bossert, D.; Thippeswamy, R.; Zharnikov, M.; Mayor, M.; Samorì, P., Multiscale Charge Injection and Transport Properties in Self-Assembled Monolayers of Biphenyl Thiols with Varying Torsion Angles. Chem. Eur. J. 2012, 18, 10335-10347.

47. Pasquier, A. D.; Miller, S.; Chhowalla, M., On the Use of Ga-in Eutectic and Halogen Light Source for Testing P3ht-Pcbm Organic Solar Cells. Sol. Energy Mater. Sol. Cells 2006, 90, 1828-1839.

48. Lilly, G. D.; Whalley, A. C.; Grunder, S.; Valente, C.; Frederick, M. T.; Stoddart, J. F.; Weiss, E. A., Switchable Photoconductivity of Quantum Dot Films Using Cross-Linking Ligands with Light-Sensitive Structures. J. Mater. Chem. 2011, 21, 11492-11497.

49. Simmons, J. G., Generalized Formula for the Electric Tunnel Effect between Similar Electrodes Separated by a Thin Insulating Film. J. Appl. Phys. 1963, 34, 1793-1803.

50. Jiang, L.; Yuan, L.; Cao, L.; Nijhuis, C. A., Controlling Leakage Currents: The Role of the Binding Group and Purity of the Precursors for Self-Assembled Monolayers in the Performance of Molecular Diodes. J. Am. Chem. Soc. 2014, 136, 1982-1991.

51. Miller, C. W.; Li, Z.-P.; Åkerman, J.; Schuller, I. K., Impact of Interfacial Roughness on Tunneling Conductance and Extracted Barrier Parameters. Appl. Phys. Lett. 2007, 90, 043513.

52. Simeone, F. C.; Yoon, H. J.; Thuo, M. M.; Barber, J. R.; Smith, B.; Whitesides, G. M., Defining the Value of Injection Current and Effective Electrical Contact Area for Egain-Based Molecular Tunneling Junctions. J. Am. Chem. Soc. 2013, 135, 18131-18144. 